\title{
Alacizumab Pegol
}

National Cancer Institute

\section{Source}

National Cancer Institute. Alacizumab Pegol. NCI Thesaurus. Code C61438.

A pegylated, cross-linked, humanized divalent-Fab' antibody fragment directed against vascular endothelial growth factor receptor-2 (VEGFR-2) with potential antiangiog enic and antitumor activities. Alacizumab pegol binds to and inhibits VEGFR-2, which may inhibit ang iogenesis and tumor cell proliferation. Multivalent Fab' antibody fragments may exhibit improved retention and internalization properties compared to their parent IgGs. 